$\Rightarrow$ PERCEPTION

\section{About faces}

Many aspects of primate social interactions rely on the ability to distinguish between the faces of different individuals. Two recent studies now provide insights into the neural mechanisms of face perception in non-human primates.

Previous work had identified a group of neurons in the macaque temporal lobe - the middle face patch - that are selectively responsive to faces. Freiwald et al. used single-cell recordings to determine how these neurons

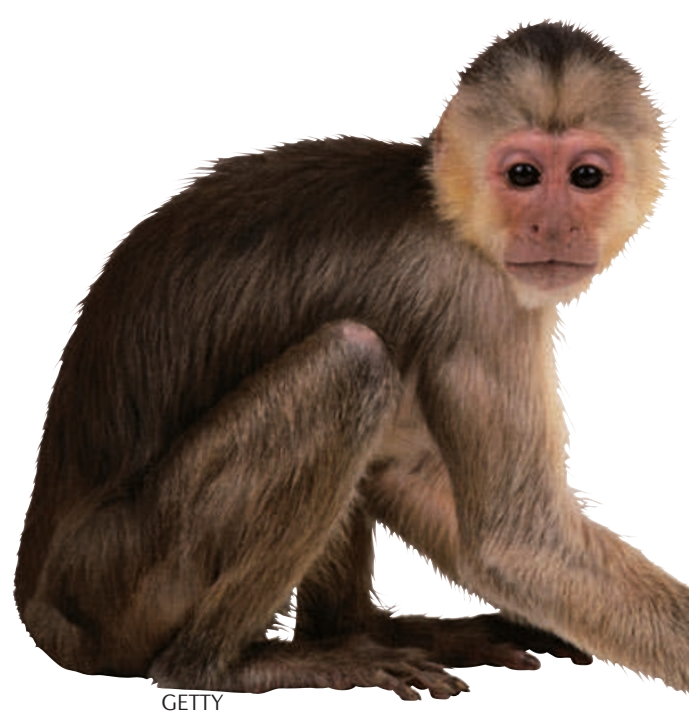

contribute to the representations of faces. They examined responses to cartoon faces consisting of 7 features (hair, face outline, eyes, irises, eyebrows, mouth and nose), the shape and distribution of which varied across 19 different dimensions - such as the distance between the eyes or the slant of the eyebrows allowing the authors to examine the selectivity and tuning of the neurons to different facial characteristics.

Individual neurons in the middle face patch responded to between one and four face parts. Furthermore, each neuron's firing rate was modified by different stimulus dimensions - each neuron was tuned to an average of three stimulus dimensions. A high proportion of neurons were tuned to dimensions related to the eyes or facial layout, whereas only a few represented dimensions related to the nose and mouth. Thus, individual neurons in the middle face patch encoded different aspects of the face.

Next, the authors asked whether the presence of a whole face was important. Although no cell required a whole face to fire, the amplification of the tuning curves and the neurons' firing rates were larger when the whole face was present. Similarly, the orientation

of the face was important: as well as an overall decrease in tuning there was a loss of tuning to eyebrow parameters and a corresponding gain in tuning to mouth parameters when the faces were inverted. This suggests that the features are analysed by comparison with an upright face template.

In their paper, Adachi et al. also considered the effect of face orientation on perception. A well-known finding in humans - the 'Thatcher effect' - is a dramatic reduction in our ability to identify differences between two faces when the faces are inverted. The authors showed that rhesus monkeys also exhibit the Thatcher effect: the monkeys spent longer looking at an altered version of a face to which they had previously been habituated (an indication of their ability to perceive the difference between the faces) when the faces were presented upright than when they were inverted, confirming that rhesus monkeys use a similar processing strategy to humans.

These studies cast light on the mechanisms of facial processing in non-human primates and indicate that certain factors, such as the importance of feature orientation, are conserved across species.

Katherine Whalley

ORIGINAL RESEARCH PAPERS Freiwald, W. A Tsao, D. Y. \& Livingstone, M. S. A face feature space in the macaque temporal lobe. Nature Neurosci. 9 Aug 2009 (doi:10.1038/nn.2363) Adachi, I., Chou, D. P. \& Hampton, R. R. Thatcher effect in monkeys demonstrates conservation of face perception across primates. Curr. Biol. 19 1270-1273 (2009) 